\title{
Bacterias ácido lácticas de bebidas vegetales fermentadas: Producción de metabolitos y propiedades antimicrobianas.
}

\author{
Lactic acid bacteria from fermented vegetable drinks: Production of metabolites \\ and antimicrobial properties. \\ Liliana Lugo-Zarate ${ }^{a}$, Nelly del Socorro Cruz-Cansino ${ }^{b}$, Alicia Cervantes-Elizarrarás ${ }^{c}$, Luis \\ Delgado-Olivares ${ }^{d}$, José Alberto Ariza-Ortega ${ }^{e}$, Quinatzin Yadira Zafra-Rojas ${ }^{f}$.
}

\begin{abstract}
:
Fruit and vegetable juices fermented with Lactic Acid Bacteria (LAB) are products that due to the structural changes caused during the fermentation process, have gained relevance within functional foods, because, in addition to the specific nutritional characteristics of the fruit such as its antioxidant content, which in addition to helping in the treatment of non-communicable diseases characterized by the presence of a state of oxidative stress, also have antimicrobial properties. BAL are microorganisms that when consumed help on intestinal colonization, promoting balance, as well as have effects associated with immunity. Another relevant application of fermented juices is the synthesis of antimicrobial compounds such as bacteriocins, which extends the possibility of using these products in the pharmaceutical area, is known to have effectiveness against resistant pathogenic microorganisms. The consumption of these products aims to promote the adequate contribution of antioxidant, achieve a balance in the microbiota and at the same time reduce microbiological resistance to drugs.
\end{abstract}

Keywords:

Fermentation, Lactic Acid Bacteria, Antioxidants, Bacteriocins, Antimicrobial Activity

Resumen:

Los jugos de frutas y hortalizas fermentados con Bacterias Ácido Lácticas (BAL) son productos que por los cambios estructurales ocasionados durante el proceso de fermentación, han ganado relevancia dentro de los alimentos funcionales, ya que, además de las características nutricionales propias del fruto como su contenido de antioxidantes, que además de coadyuvar en el tratamiento de enfermedades no transmisibles caracterizadas por la presencia de un estado de estrés oxidativo, presentan propiedades antimicrobianas. Las BAL son microorganismos que al consumirlos ayudan sobre la colonización intestinal, promoviendo equilibrio, además tienen efectos asociados a la inmunidad. Otra aplicación relevante de los jugos fermentados es la síntesis de compuestos antimicrobianos como las bacteriocinas, que extiende la posibilidad de utilizar estos productos en el área farmacéutica, se conoce que tienen efectividad contra microorganismos patógenos resistentes. Con el consumo de estos productos se quiere promover el aporte adecuado de antioxidante, lograr un equilibrio en la microbiota y al mismo tiempo disminuir las resistencias microbiológicas a los fármacos.

Palabras Clave:

Fermentación, Bacterias Ácido Lácticas, Antioxidantes, Bacteriocinas, Actividad antimicrobiana

\footnotetext{
a Universidad Autónoma del Estado de Hidalgo, https://orcid.org/0000-0002-4772-8686, Email: lilianalugozarate@outlook.com

b Autor de Correspondencia, Universidad Autónoma del Estado de Hidalgo, https://orcid.org/0000-0002-6771-3684, Email: ncruz@uaeh.edu.mx

c Universidad Autónoma del Estado de Hidalgo, https://orcid.org/0000-0002-1432-2882, Email: alicia_cervantes@uaeh.edu.mx

d Universidad Autónoma del Estado de Hidalgo, https://orcid.org/0000-0002-3506-8393, Email: ldelgado@uaeh.edu.mx

e Universidad Autónoma del Estado de Hidalgo, https://orcid.org/0000-0002-2163-4593, Email: ariza_ortega@yahoo.com.mx

f Universidad Autónoma del Estado de Hidalgo, https://orcid.org/0000-0002-5295-9972, Email: quinatzin_zafra@uaeh.edu.mx 


\section{Introducción}

La alta demanda de productos alimentarios que otorguen propiedades benéficas, más allá de su propia composición, ha llevado a la industria a buscar alternativas con el uso de alimentos que cumplan con estas expectativas.

Uno de ellos son las frutas y hortalizas que se caracterizan por su contenido en vitaminas y antioxidantes con alta actividad antioxidante ${ }^{1}$ y propiedad antimicrobiana ${ }^{2}$. Los jugos o zumos son productos que pueden elaborarse a base de estos ingredientes, sin embargo, son alimentos perecederos susceptibles a la fermentación. Este proceso de fermentación ha sido utilizado en este tipo de alimentos para mejorar su conservación, y determinar los cambios sobre las propiedades nutricionales de la actividad metabólica y enzimática de los microorganismos sobre el producto fermentado ${ }^{3}$. De los procesos fermentativos, el más estudiado es el del ácido láctico producido por las BAL, éstas son las más utilizadas en la fermentación de jugos y hortalizas debido a su inocuidad y beneficios a la salud humana ${ }^{4}$.

Recientemente se ha informado que el uso excesivo de antibióticos ha generado resistencia a los medicamentos antibacterianos, el cual es un problema de salud pública de acuerdo con la Organización Mundial de la Salud $(\mathrm{OMS})^{5}$. El descubrimiento de los antimicrobianos fue importante, ya que permitió que la calidad y la esperanza de vida aumentaran ${ }^{6}$, sin embargo, en la actualidad la alimentación ha cambiado, ocasionando susceptibilidades a la población a diversas enfermedades, entre ellas las causadas por microorganismos patógenos ${ }^{7,8}$. Uno de los procesos por los cuales se quiere lograr la síntesis de compuestos antimicrobianos es la fermentación, y la industria alimentaria se ha centrado en los probióticos, que se definen como microorganismos vivos que confieren beneficios a la salud del huésped ${ }^{9}$. La nueva gama de productos antimicrobianos son los jugos fermentados a partir de BAL que producen metabolitos que pueden sustituir a antibióticos, con el propósito de disminuir los efectos adversos ${ }^{10}$.

La presente revisión tiene como objetivo, recopilar información sobre las propiedades antioxidantes y antimicrobianas de bebidas de frutas y hortalizas fermentadas con BAL, así como conocer acerca de los metabolitos que otorgan esta propiedad.

\section{Bebidas vegetales}

Una bebida es una sustancia líquida, que puede estar compuesta de diferentes ingredientes ${ }^{11}$, se conocen diferentes tipos como el agua, leches, procesados (refrescos, acoholes, vinos y jugos), artesanales como el tepache, vegetales como cereales, frutas y hortalizas.

Como definición, las bebidas vegetales o no lácteas son elaboradas con agua e ingredientes vegetales sin proteína animal ${ }^{12}$, y se elaboran a partir de alimentos como leguminosas (soya, cacahuate, chícharo, frijol), oleaginosas (almendra y avellanas, ajonjolí y cáñamo), plantas aceitosas (palma del coco), cereales (maíz, trigo, avena, arroz, kamut, espelta), pseudocereales (amaranto, quinua, alforfón) y sedges (almendra de tierra) ${ }^{13}$. Para la elaboración de bebidas vegetales, se puede hacer uso de endulzantes como miel, azúcar o estevia y se puede elevar su contenido en grasa con aceites vegetales como el de oliva, soya, girasol, sésamo, entre otros ${ }^{12}$.

En los últimos 20 años, se ha observado un aumento en el consumo de estas bebidas, principalmente la de soya en las regiones de Norteamérica, Latinoamérica, Europa y Asia ${ }^{14}$.

Otro tipo de bebidas vegetales son las elaboradas a base de frutas y hortalizas, las cuales pueden ser de una sola especie o diferentes, sin la adición de edulcorantes calóricos ${ }^{15}$.

Las bebidas vegetales se han consumido a pequeña escala desde el inicio de las civilizaciones ${ }^{16}$. Su creciente popularidad se debe a la alta prevalencia de enfermedades no transmisibles o el creciente número de personas con alergia a la proteína de la leche y lactosa ${ }^{17}$. Integrar este producto a la dieta posee efectos positivos por su contenido en vitaminas, fibra, bajo en colesterol, compuestos bioactivos y el contenido proteico de alta calidad otorgado por los cereales ${ }^{18}$.

Comparando a los cereales y oleaginosas con los frutas y hortalizas, estos últimos son perecederos por su alto contenido en agua (70-95\%), por una mayor tasa de respiración, lo cual favorece el crecimiento de microorganismos ${ }^{19}$ ocasionado su descomposición. Las frutas y hortalizas en fresco son alimentos poco aprovechados por la población, ya que se estima un desperdicio anual de 40 a $50 \%$ de la producción total ${ }^{20}$. Por lo tanto, la industria alimentaria busca elaborar productos que promuevan la preservación y aprovechamiento de este grupo de alimentos y una forma de hacerlo es mediante la elaboración de jugos. Estos jugos se destacan por su composición nutrimental en carbohidratos, vitaminas y minerales de forma natural.

\section{Composición nutricional de jugos de frutas y hortalizas}

Los jugos o zumos, se obtienen de frutas y hortalizas sanas, maduras, frescas o conservadas por el frío, que tengan el color, sabor, aroma característico, igualmente pueden obtenerse de un concentrado ${ }^{21}$, el cual se obtiene de la eliminación de agua para aumentar al menos 50\% de sólidos solubles totales ${ }^{22}$. 
Los jugos de fruta pueden ser clarificados o no, deben ser sometidos a un tratamiento que garantice su inocuidad y conservación, además no debe contener piel ni semillas, se puede añadir pulpa del mismo fruto ${ }^{22}$.

Los jugos pueden suplir a una fruta fresca en la dieta mientras no sean añadidos con azúcares, y tengan valores equivalentes en composición nutrimental con las frutas y hortalizas de origen ${ }^{21,22}$.

La composición de las frutas y hortalizas está representada mayormente por agua (75-90\%), seguido de hidratos de carbono (hortalizas $3-20 \%$ y frutas $5-18 \%$ ), proteínas (hortalizas $1-5 \%$ y frutas $0.1-1.5 \%$ y los lípidos que son minoritarios (hortalizas $0.1-0.9 \%$ y frutas 0.1 $0.5 \%$ ). Son fuente rica de vitaminas, minerales, pectina, fibras, ácidos orgánicos y compuestos de interés como los antioxidantes, los cuales son característicos de este grupo de alimentos ${ }^{23}$.

Por lo anterior, los jugos pueden ser un alimento que ofrezca beneficios a la salud, atribuibles a su composición, además se caracteriza por compuestos no nutritivos (como los antioxidantes) pero con funciones importantes en el organismo.

Propiedad antioxidante de jugos de frutas y hortalizas Los compuestos antioxidantes tienen alta eficacia contra el estrés oxidativo, este se produce cuando hay una capacidad insuficiente del sistema endógeno antioxidante biológico y se producen aniones superóxido, el radical generado con mayor frecuencia que daña la célula y $\mathrm{ADN}^{24}$.

Los jugos de frutas y hortalizas tienen compuestos con gran actividad antioxidante, estos además otorgan color, olor y sabor al alimento, algunos compuestos como el ácido ascórbico son indicadores de calidad en los jugos ${ }^{25}$, en la Tabla 1 se resume el contenido antioxidante de diferentes jugos.

El tomate que es fuente de carotenoides principalmente licopeno, este es un pigmento natural liposoluble que da el color rojo y naranja a frutas y hortalizas ${ }^{26}$, se le atribuyen principalmente efectos endoteliales, antiateroscleroticos, antiinflamatorios, antihipertensivos, antiapoptóticos entre otros ${ }^{27}$. Otra fuente de carotenoides es a partir del jugo de zanahoria, este tipo de antioxidante tiene efectos importantes sobre cáncer, afecciones oculares, cutáneas, etc. ${ }^{28}$.

Otro antioxidante de importancia es el ácido ascórbico, el cual se encuentra en jugos de tomate, piña, zanahoria, pera, carambolo, guanábana, entre otros (Tabla 1). El ácido ascórbico es compuesto abundante en los alimentos ${ }^{29}$ sin embargo, es sensible a la luz, calor, concentración de oxígeno, $\mathrm{pH}$, a pesar de ello es un compuesto aclamado por ser potente eliminador de radicales libres ${ }^{30}$, en combinación con vitaminas $A$ y $B$, disminuye la gravedad de pacientes que padecen artritis reumatoide ${ }^{31}$, elimina especies reactivas de oxígeno durante la actividad sináptica y el metabolismo neuronal, su desequilibrio ocasiona enfermedades neurodegenerativas como Alzheimer, Parkinson, esclerosis múltiple ${ }^{32}$.

Los compuestos fenólicos son otros componentes importantes de los frutas y hortalizas, que igualmente se encuentran en altas concentraciones en los jugos y se caracterizan por sus propiedades sobre el estrés oxidativo, enfermedades cardiovasculares, cáncer, diabetes tipo 2 y osteoporosis ${ }^{33}$. La mayoría de los fenoles no son absorbibles e interactúan continuamente con el epitelio gastrointestinal y la microbiota, ésta los metaboliza para contribuir positivamente con los enterocitos del colon $^{34,35}$.

Tabla 1. Antioxidantes en jugos de frutas y hortalizas

\begin{tabular}{cc} 
Jugo & \multicolumn{1}{c}{ Antioxidante } \\
\hline Tomate & Carotenoides \\
& $131.5 \mathrm{mg} / 100 \mathrm{~g} \mathrm{bs}{ }^{36}$ \\
& Licopeno \\
& $118 \mathrm{mg} / 100 \mathrm{~g} \mathrm{bs}$ \\
& $30.68 \mathrm{ug} / \mathrm{g}^{37}$ \\
& Ácido ascórbico \\
& $274 \mathrm{mg} / 100 \mathrm{~g} \mathrm{bs}{ }^{36}$ \\
& $395.52 \mathrm{mg} / 100 \mathrm{~mL}^{38}$ \\
& Fenoles totales \\
& $398.67 \mathrm{EAG} \mathrm{ug}^{36} \mathrm{~g}^{36}$ \\
& Fenoles totales \\
& $85.73 \mathrm{mg} \mathrm{EAG} / \mathrm{g} \mathrm{bs}^{39}$ \\
& $185 \mathrm{mg} \mathrm{EAG} / 100 \mathrm{~mL}^{40}$ \\
Piña & Antocianinas totales \\
& $0.86 \mathrm{mg} / 100 \mathrm{~mL}^{39}$ \\
& $\beta$-carotenos \\
& $0.95 \mathrm{mg} / 100 \mathrm{ml}^{41}$ \\
& Ácido ascórbico \\
& $45.84 \mathrm{mg} / 100 \mathrm{ml}^{39}$
\end{tabular}


Carotenoides

$1777.5 \mathrm{ug} / 100 \mathrm{~mL}^{44}$

$572.7 \mathrm{mg} / \mathrm{L}^{45}$

$\beta$-carotenos

$9.34 \mathrm{mg} / 100 \mathrm{ml} 41$

\begin{tabular}{|c|c|}
\hline Betabel & 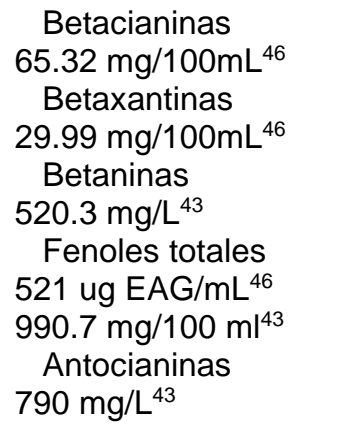 \\
\hline Pera & $\begin{array}{c}\text { Fenoles totales } \\
345.8 \mathrm{ug} \text { EAG/g } / \mathrm{g}^{47} \\
357 \mathrm{ug} \text { EAG } / \mathrm{g}^{48} \\
\text { Flavonoides } \\
179.93 \mathrm{ug} \mathrm{EC} / \mathrm{g}^{48} \\
\text { Ácido ascórbico } \\
4.14 \mathrm{mg} / 100 \mathrm{~mL}^{47} \\
3.56 \mathrm{mg} / 100 \mathrm{~mL}^{48}\end{array}$ \\
\hline Carambolo & $\begin{array}{l}\text { Ácido ascórbico } \\
24.17 \mathrm{mg} / 100 \mathrm{~mL}^{49} \\
\text { Fenoles totales } \\
540 \mathrm{mg} \text { EAG/100 } \mathrm{mL}^{49} \\
\text { Flavonoides } \\
2.56 \mathrm{mg} \text { EQ/100 } \mathrm{mL}^{49}\end{array}$ \\
\hline Guanábana & $\begin{array}{l}\text { Ácido ascórbico } \\
44.76 \mathrm{mg} / 100 \mathrm{~g}^{50} \\
\text { Fenoles totales } \\
187.95 \mathrm{mg} / 100 \mathrm{~g}^{50}\end{array}$ \\
\hline Granada & $\begin{array}{l}\text { Fenoles totales } \\
80.60 \mathrm{mg} \text { EAG/g bs } \\
\text { Antocianinas } \\
15.98 \mathrm{mg} / 100 \mathrm{~mL}^{39}\end{array}$ \\
\hline $\begin{array}{l}\text { Naranja con } \\
\text { apio }\end{array}$ & $\begin{array}{l}\text { Fenoles totales } \\
247 \mathrm{mg} \text { EAG/100 mL } \\
\text { Flavonoides } \\
1.56 \mathrm{mg} \text { EQ/100 mL } \\
\text { Ácido ascórbico } \\
37.78 \mathrm{mg} \mathrm{AA} / 100 \mathrm{~mL}^{51}\end{array}$ \\
\hline $\begin{array}{c}\text { Manzana } \\
\text { (comercial) }\end{array}$ & $\begin{array}{l}\text { Fenoles totales } \\
507 \mathrm{mg} / \mathrm{L}^{52} \\
\text { Fenoles totales } \\
438 \mathrm{mg} \mathrm{EAG} / \mathrm{L}^{53} \\
\text { Flavonoides } \\
196 \mathrm{mg} \mathrm{EC} / \mathrm{L}^{53}\end{array}$ \\
\hline Melón & $\begin{array}{l}\text { Fenoles totales } \\
182.27 \text { ug EAG/mL } \\
\text { Ácido ascórbico }\end{array}$ \\
\hline
\end{tabular}

$23.84 \mathrm{mg} \mathrm{AA} / 100 \mathrm{~mL}^{54}$

bs: base seca; EAG: Equivalentes de Ácido Gálico; EC: Equivalentes de Catequinas; $E Q$ : Equivalentes de Quercetina; AA: Ácido ascórbico.

Otro de los compuestos antioxidantes son las antocianinas, catequinas, taninos, ácido gálico y elágico, estos están presentes en la granada, con efectos antimicrobianos ${ }^{55}$, los polifenoles de jambolán ${ }^{56}$ o las antocianinas de arándano, donde la cianidina y malvidina son los compuestos más abundantes ${ }^{57}$.

\section{Propiedades antimicrobianas de los jugos}

Los antioxidantes son agentes naturales presentes en el alimento con efectos antimicrobianos, ya que pueden retardar o inhibir el crecimiento de agentes patógenos ${ }^{58,59}$ por inhibición enzimática ${ }^{60}$, inhibición de síntesis de ácidos nucleicos ${ }^{61}$, inhibición del metabolismo energético ${ }^{62}$, disminución de la unión celular, cambio en la permeabilidad de la membrana y alteración funcional de la membrana citoplasmática ${ }^{63,64}$. Sin embargo, de algunos antioxidantes como los polifenoles, no se conoce el mecanismo de acción antimicrobiano ${ }^{65}$.

La propiedad antimicrobiana de los antioxidantes en jugos elaborados a partir de frutas y hortalizas, depende mayormente de los compuestos fenólicos, como en concentrados de jugos de mandarina, uva, limón y lima, los cuales contienen flavonoides, con poder antimicrobiano actuando contra bacterias gram positivas (Staphylococcus aureus y Enterococcus faecalis), gram negativas (Escherichia coli y Salmonella spp.) y hongos (C. albicans, A. nigery Penicillum spp.), teniendo una zona de inhibición de 8 a $26 \mathrm{~mm}$, de 4 a $25 \mathrm{~mm}$ y de $0-24 \mathrm{~mm}$, respectivamente ${ }^{66}$. Los compuestos fenólicos del jugo de lima tienen efecto inhibidor contra Pseudomonas aeruginosa, S. aereus y $E$. faecalis, sin embargo, los antibióticos utilizados como referencia (rifampicina, oxacilina y ampicilina) tienen mejor efecto sobre estos microorganismos, ya que la zona de inhibición de los antibióticos es entre 12.1-23.8 mm y del jugo de lima es entre 9.1-11.2 $\mathrm{mm}^{67}$.

Además de las propiedades antimicrobianas de los antioxidantes, los jugos de frutas y hortalizas son susceptibles a la fermentación, principalmente por su alto contenido en carbohidratos, algunas fermentaciones no controladas pueden ser inaceptables por el consumidor, sin embargo, cuando la fermentación es monitoreada y controlada tiene efectos deseables ${ }^{68}$.

\section{Fermentación}


La fermentación se define como el proceso metabólico celular que produce sustancias orgánicas simples, como etanol, ácido láctico y butírico a partir de carbohidratos, como la lactosa, sacarosa o fructosa ${ }^{69}$. Se da por acción de los microorganismos propios del alimento, por ingredientes alimentarios añadidos o del medio ambiente y por cultivos iniciadores ${ }^{70}$. El uso de estos es la forma más común de la fermentación sumergida que es utilizada en los jugos ${ }^{71}$. Además, es usada como método de conservación, mejora de sabor, busca convertir alimentos perecederos e indigeribles en alimentos agradables y bebidas con un valor agregado y estables ${ }^{72,73}$.

Los principales microorganismos fermentadores son las levaduras, las bacterias propiónicas, las metanogénicas y las $\mathrm{BAL}^{74}$. Las BAL llevan a cabo la fermentación láctica en diversos jugos como el de manzana y piña con $L$. mesenteroides, $W$. cibaria y $L$. pseudomesenteroides68.

Las vías participantes en el desarrollo de sabor en los alimentos fermentados son la glucólisis, lipólisis y proteólisis ${ }^{75}$, por lo cual una bebida fermentada debe tener un sustrato adecuado, y microorganismos apropiados en condiciones ambientales ideales (temperatura, $\mathrm{pH}$, humedad $)^{76}$. Las BAL son los microorganismos más utilizados para los procesos de fermentación ${ }^{77}$.

\section{Bacterias ácido lácticas (BAL)}

Las BAL son un grupo de microorganismos gram positivos, con forma microscópica de cocos o bacilos que no forman esporas ${ }^{78}$. Crecen a temperatura entre $5 \mathrm{y}$ $45^{\circ} \mathrm{C}, \mathrm{pH}$ óptimo de 5.5 a 6.5 y en hábitats con un rico suministro de alimento ${ }^{79}$ que incluye aminoácidos, péptidos, nucleótidos, vitaminas, minerales, ácidos grasos y carbohidratos ${ }^{80}$.

Las BAL se aíslan de diferentes fuentes, principalmente alimentarias, como el kimchi, del cual se aísla al Leuconostoc mesenteroides y Lactobacillus plantarum, estos microorganismos son importantes para su fermentación natural sin la adición de un cultivo iniciador $^{81}$. A partir de leche de camello cruda se identifica al Lactococcus lactis, L. plantarum, Lactobacillus reuteri, entre otros microorganismos pertenecientes a $B A L^{82}$. En frutas como las uvas, se encuentra el Pediococcus pentosaceus y $L$. plantarum ${ }^{83}$ y en tunas verdes al Fructobacillus fructuosus y L. plantarum ${ }^{84}$; mientras que la papaya, tomate y repollo tienen microorganismos del género Fructobacillus, Lactobacillus, Lactococcus, Leuconostoc y Weissella (85). En productos no lácteos (tomate, piña y zanahoria) y lácteos (quesos brasileño, parmigiano Reggiana y grana padano) se aísla al $L$. plantarum, L. rhamnosus y $L$. case ${ }^{\beta 6}$, mientras que en heces de bebés de 2 semanas de edad, sanos y con lactancia materna exclusiva se encuentra a $L$. reuteri, Lactobacillus gasseri y Lactobacillus case ${ }^{87}$.
Dentro de las BAL que se identifican comúnmente se encuentran las del género Lactobacillus, estos microorganismos forman parte del microbiota saludable del ser humano ${ }^{10,88}$, algunos se emplean como probióticos por su sobrevivencia, resistencia a condiciones gástricas y adherencia celular ${ }^{89}$.

Las bacterias ácido lácticas se dividen en homofermentativas y heterofermentativas ambas producen ácido láctico, pero la formación de alcohol y $\mathrm{CO} 2$ es solo en las segundas ${ }^{90}$. Principalmente se caracterizan por la producción de ácido láctico, como producto final catabólico y otros ácidos orgánicos como el ácido acético y propiónico ${ }^{78}$. Durante la fermentación en caldos de infusión de pescado a partir de BAL de géneros Lactococcus, Lactobacillus, Streptococcus, Leuconostocy Pediococcus, se producen diferentes ácidos orgánicos como el fórmico, láctico, acético, succínico, propiónico y butírico, sin embargo, el que en menor concentración se produce es el acético ${ }^{91}$.

Los ácidos orgánicos evitan el deterioro y el crecimiento de patógenos, fomentando la conservación de los alimentos como verduras, frutas, productos lácteos, entre otros ${ }^{79}$.

Los metabolitos producidos tienen propiedades benéficas como antibióticos, anticancerígenos ${ }^{92,93}$, antimicrobianos ${ }^{79,94}$ o con propiedades antioxidantes ${ }^{95}$. Estos microorganismos, administrados en cantidades adecuadas tiene un beneficio en la salud del huésped, por esta razón se aíslan las BAL para crear cultivos iniciadores utilizados para comenzar un proceso fermentativo en diferentes productos alimentarios como los jugos.

\section{Fermentación de jugos por BAL}

La fermentación de jugos a partir de BAL, puede ser de manera espontánea (microorganismos propios del fruto) 0 controlada (cultivo iniciador) ${ }^{96}$, y pueden prolongar la vida útili $^{97}$, mejora el sabor, textura y potencia efectos probióticos ${ }^{98}$. Es por ello que estos productos se han popularizado en los últimos años.

El proceso fermentativo tiene diferentes propósitos como el de mejorar las características sensoriales, incrementar y sintetizar compuestos bioactivos de interés, otorgar un producto de alto valor biológico, así como el tener un producto adecuado para personas con alergias o intolerancias ${ }^{96}$ como se mencionó anteriormente.

Durante la fermentación de jugos, se detecta la producción de diversos compuestos como los volátiles, en el jugo de manzana fermentado con 4 diferentes BAL ( $L$. acidophilus, L. rhamnosus, L. casei y L. plantarum), se producen 53 compuestos volátiles después de la fermentación, compuestos comos acetaldehídos y cetonas, y durante el proceso por $L$. acidophilus incrementan los componentes aromáticos (e-etilhexanol, 
3-metil-1-butanol, 2-metil-1-butanol y acetato de etilo) ${ }^{99}$. En jugo de azufaifo fermentado con $L$. plantarum, también se producen más compuestos aromáticos y menos orgánicos de azufre, dentro de las características del jugo es que su sabor es ácido, menos amargo y astringente, en comparación con los cultivos de $L$. casei y E. faecium ${ }^{100}$. En jugo de mango fermentado con cultivos autóctonos de Leuconostoc mesenteroides, también hay síntesis de ácidos orgánicos y diacetilo ${ }^{101}$. Por otro lado, durante la fermentación de jugo de saúco con diferentes aislados de cepas Lactobacillus de origen lácteo, se producen ácidos fenilácticos, se modifica el contenido de ácidos hidroxicinámicos y aumentan los compuestos fenólicos ${ }^{102}$.

Otra característica de la fermentación de jugos, es el contenido de antioxidantes, se sabe que el contenido de fenoles puede mantenerse, aumentar o disminuir dependiendo del sustrato y del microorganismo, así como de los compuestos agregados intencionalmente (enzimas y carbohidratos), los cuales llegan a modificar el proceso de fermentación.

En jugo de anacardo fermentado con $L$. casei, $L$. plantarum y L. acidophilus, el ácido ascórbico aumenta a las 12 horas para posteriormente disminuir, los fenoles totales no presentan cambios y los taninos condensados aumentan ligeramente ${ }^{103}$. En el jugo de tuna verde fermentado con $L$. plantarum, se mantiene el contenido de fenoles totales con $565.1 \mathrm{mg} \mathrm{EAG} / \mathrm{mL}^{104}$; existe un aumento de antocianinas y polifenoles en jugo de cereza fermentado con un cultivo un iniciador de $L$ plantarum y $L$. rhamnosus, microorganismos aislados de productos lácteos, mientras que el mismo jugo fermentado con BAL aisladas de productos vegetales, aumenta mayormente la producción de ácidos fenilacéticos ${ }^{105}$.

En jugo de mora fermentado con 3 diferentes BAL (L. plantarum, L. acidophilus y L. paracasei), aumentan las antocianinas, los fenoles y los flavonoides en comparación con el jugo sin fermentar, y el jugo inoculado con $L$. plantarum tiene mayor concentración de fenoles totales (695.51 ug/mL), la elaborada con L. paracasei tiene más antocianinas (307.56 ug/mL) y con respecto a los flavonoides la mejor es la de L. acidophilus (851.73 $\mathrm{ug} / \mathrm{mL})^{106}$.

Neffe-Skocinska en $2018^{107}$ menciona que las BAL coadyuvan en la salud gastrointestinal de la persona que los consume, e indica que este tipo de alimentos fermentados, debe contener un nivel mínimo terapéutico de microorganismos probióticos de al menos $10^{6} \mathrm{UFC} / \mathrm{g}$ durante la vida útil del producto. Algunas propiedades otorgadas por el jugo de betabel co-cultivado con $L$. acidophilus y L. plantarum, es la actividad citotóxica contra células humanas de cáncer de hígado (Hep G2) ${ }^{108}$. La administración de jugo de tuna verde fermentado con $L$. plantarum en ratones obesos provoca la disminución del peso, mejora la resistencia a la insulina, la hiperglucemia y la hiperlipemia ${ }^{104}$, además tienen propiedades antimicrobianas que confieren las BAL.

Actividad antimicrobiana de jugos fermentados con BAL La actividad antimicrobiana consiste en inhibir el crecimiento o eliminación de un microorganismo patógeno, esta propiedad ha sido descrita en jugos fermentados por BAL, y son diferentes los compuestos y mecanismos que le confieren esta característica ${ }^{109}$.

La modificación de compuestos durante la fermentación ocurre en los jugos, como es el caso de los antioxidantes los ácidos orgánicos, diacetilo, bacteriocinas y otros, su síntesis depende de la cepa y el sustrato.

En fermentaciones espontáneas hay menor control sobre los microorganismos que pueden crecer, un ejemplo de ello es el jugo de zanahoria, que tiene mayor cantidad de BAL en comparación con las enterobacterias, 8 de 20 cepas identificadas por secuenciación pertenecen a Leuconostoc y Lactobacillus ${ }^{110}$; igualmente en la fermentación espontánea de jugo de col rizada se encuentra mayor conteo de BAL y un nulo crecimiento de E. coli y Salmonella spp., la actividad antimicrobiana mejora durante la fermentación contra diferentes cepas patógenas $(E$. coli, S. enteritidis, $Y$. enterocolitica, $P$. aeruginosa, entre otras) $)^{4}$.

El jugo de uva roja fermentado con BAL aisladas de kombucha, presenta actividad antimicrobiana contra $E$. coli, P. aeruginosa, S. aureus, E. fecalis, B. cereus y $S$. epidermidis, similar al antibiótico cloranfenicol, esta propiedad se atribuye a la producción de ácido acético ${ }^{111}$; mientras que en el jugo de col rizada fermentado con BAL aisladas de un fermentado espontáneo ( $L$. plantarum, $W$. hellenica, W. cibaria, L. brevis, P. pentosaceus, entre otras), se le atribuye su actividad antimicrobiana a la alta actividad antioxidante, las zonas de inhibición que presenta sobre $S$. aureus son de 10-60 mm, con Salmonella enterica son de 10-84 $\mathrm{mm}$ y con E. coli son de 7-37 $\mathrm{mm}^{112}$; el ácido láctico que se produce en el jugo de manzana fermentado con $L$. plantarum y $S$. thermophilus, tiene actividad antimicrobiana contra E. coliy S. aureus ${ }^{113}$.

El uso de microorganismos probióticos promete ser un buen coadyuvante en el tratamiento de las infecciones causadas por microorganismos patógenos, debido a los efectos demostrados su consumo tiene resultados positivos en afecciones que van desde diarrea, colitis por Clostridium, infecciones por Helicobacter pylori, cáncer, etc. ${ }^{109}$.

Los mecanismos de acción antimicrobiana de los probióticos a nivel molecular se desconocen, pero la exclusión competitiva, la competencia por nutrientes 0 modulación del sistema inmune y la síntesis de compuestos como las bacteriocinas son algunos de los mecanismos por los que se le confiere esa propiedad ${ }^{114}$. 
que además de tener efectos positivos sobre el producto, la microbiota intestinal se ve beneficiada.

\section{Actividad antimicrobiana por bacteriocinas}

Las bacteriocinas son compuestos de naturaleza peptídica, sintetizadas en el ribosoma y originadas durante el metabolismo de las BAL, son considerados compuestos seguros y antimicrobianos que promueven la biopreservación ${ }^{115}$, son un rasgo probiótico importante que promueve el equilibrio, colonización y persistencia bacteriana en el tracto gastrointestinal ${ }^{116}$, las bacteriocinas consumidas sirven como terapia para eliminar microorganismos resistentes ${ }^{117}$. Las condiciones óptimas de síntesis de estos metabolitos péptidos son a $37^{\circ} \mathrm{C}, \mathrm{pH}$ de 6-6.5 por 16-24 $\mathrm{h}^{118}$, aunque pueden producirse a diferentes temperaturas de $15-50^{\circ} \mathrm{C}, \mathrm{pH}$ de $4-10$ y concentraciones de $\mathrm{NaCl}$ de 5 y $8 \%$ como es el ejemplo a partir de E. faecium, $P$. acidilactici, L. plantarum, entre otros $^{119}$.

Para evaluar la actividad antimicrobiana de las bacteriocinas, se realizan extractos estériles libres de células. La actividad antimicrobiana de las bacteriocinas de L. sakei tienen contra Listeria monocytogenes es de 1067 Unidades Arbitrarias $/ \mathrm{mL}^{120}$.

Las bacteriocinas a partir de 3 aislados de BAL del jugo de lima dulce fermentado de forma espontánea, tiene efectividad contra $E$. coli, $S$. aureus, $B$. subtilis y $P$. aeruginosa, con halos de inhibición de 10-12 $\mathrm{mm}$ de 9-11 $\mathrm{mm}, 8-9 \mathrm{~mm}$ y de $6-8 \mathrm{~mm}$, respectivamente ${ }^{121}$. Las bacteriocinas producidas por L. plantarum aislado del jugo de malus fermentado, presenta actividad antimicrobiana a partir del segundo día en jugos artesanales fermentados de naranja y chicha con presencia de $E$. coli y $S$. enterica $^{122}$.

La identificación de bacteriocinas como la nisina A y lactococcins $B$ en $L$. lactis ${ }^{123}$, de pediocina PA-1 por $E$. faecium, $P$. acidilactici, $L$. plantarum, entre otros ${ }^{119}$, la lacticina 481 producida por bacterias de la especie $L$. lactis $^{124}$, son péptidos que demuestran tener efecto protector en el tracto gastrointestinal al excluir patógenos o promoviendo la colonización intestinal ${ }^{117}$. La producción de bacteriocinas de BAL aisladas de la microbiota intestinal humana tienen actividad antimicrobiana contra E. coli, S. aureus, S. entérica y L. innocua ${ }^{125}$.

Las bacteriocinas principalmente actúan sobre la membrana formando poros produciendo vaciamiento intracelular generando la pérdida de iones potasio, energía y moléculas de bajo peso molecular, como consecuencia desencadena la muerte celular ${ }^{126}$. Otra forma de actuar de las bacteriocinas es por la naturaleza anfipática que facilita su inserción a la membrana bacteriana despolarizando y causando la muerte ${ }^{127}$.
Bacterias ácido lácticas sobre microorganismos patógenos de la microbiota intestinal

Se ha generado mucho interés sobre la diversidad de la microbiota intestinal por su asociación con enfermedades $^{128}$, su composición microbiana no es estática, está en recambio desde el nacimiento ${ }^{129}$, por lo tanto las bacterias que están por mayor tiempo en el intestino, interactúan con el epitelio desempeñando un papel inmunomodulador ${ }^{130}$. El estilo de vida, la alimentación, la edad, el consumo de antibióticos, entre otros factores modifican estructuralmente la microbiota de forma aguda o crónica ${ }^{131}$.

Las bacterias de la microbiota pertenecen a diferentes géneros, los principales son Gram-negativas (Bacteriodes y Fusobacterium), Gram-positivas (Eubacterium, Bifidobacterium, Lactobacillus y Clostridium), cocos (Ruminococcus y Peptostreptococcus) y Enterobacteriaceae (E. coli, Citrobacter, Enterobacter, Proteus, Shigella, Salmonella y Yersina) ${ }^{132}$. El único microorganismo que alberga mayormente en el estómago debido a su acidez es el Helicobacter pylori, considerado un carcinógeno tipo $1^{133}$. La colonización comienza en el duodeno, y en el intestino grueso se ubica la mayor densidad microbiana ${ }^{134}$. Y la proporción de BAL en la microbiota gastrointestinal es de $1 \%$, muchas de ellas son pasajeras $^{10}$.

La microbiota intestinal mejora la inmunidad contra patógenos, actúa en una interacción directa con la luz intestinal, con la mucosa y el epitelio intestinal ${ }^{135}$, por eso su importancia en la digestión, metabolismo y función inmune. Su desequilibrio se asocia con trastornos gastrointestinales, que se pueden controlar con el consumo de microorganismos probióticos en cantidades adecuadas $^{129}$. Es por esto que diversas investigaciones se centran en la obtención de productos funcionales como las bebidas fermentadas, que al ser consumidas promuevan la colonización de BAL en la microbiota ${ }^{136}$.

Los mecanismos antimicrobianos directos desarrollados por las BAL, son por competición como la coagregación con patógenos y la adherencia competencia, por sustratos alimentarios, acumulacion de D-aminoacidos ${ }^{137}$, además de la producción de metabolitos encargados de la actividad antimicrobiana indirecta, como el peróxido de hidrógeno ${ }^{138}$.

Son pocos los jugos de frutas y hortalizas fermentados que prueban su actividad antimicrobiana en modelos in vivo o población humana, como el jugo de betabel fermentado con $L$. casei y $L$. brevis suplementado

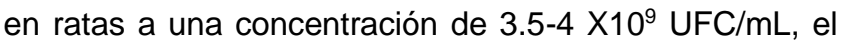
análisis de la microbiota determina una disminución logarítmica de 0.8-2.1 UFC en Enterobacteriaceae ${ }^{139}$, el consumo de un fermentado de jugo de betabel por $L$. brevis y $L$ paracasei en ratas, disminuye las concentraciones de Clostridium coccoides y 
Enterobacteriaceae ${ }^{140,}$ igualmente en jugo de arándano fermentado a partir de L. casei, mejora la comunidad microbiana en un ensayo in vitro sobre muestras fecales con la inhibición de E. coli, Enterococcus, Firmicutes y Bacteroides, aumentando los conteos de Bifidobacterium $y$ Lactobacillus en las muestras fecales ${ }^{141}$.

Lo anterior indica la importancia de las BAL en la microbiota humana, ya que promueven efectos benéficos a la salud mediante su colonización en el tracto gastrointestinal, lo que permite mantener un equilibrio sobre los microorganismos patógenos.

\section{Conclusión}

Los jugos de frutas y hortalizas prometen ser un alimento funcional por su contenido en antioxidantes, además la preservación de alimentos perecederos por el uso de BAL mediante la fermentación, es un proceso metabólico del cual se obtienen diferentes metabolitos importantes que coadyuvan a la salud humana. Uno de los efectos más conocidos es la actividad antimicrobiana que tiene un papel importante en la conservación de los alimentos y protección contra patógenos que causan enfermedades en el cuerpo humano.

\section{Referencias}

1. Płonka J, Toczek A, Tomczyk V. Multivitamin Analysis of Fruits, Fruit-Vegetable Juices, and Diet Supplements. Food Anal Methods 2012.

2. Puupponen-Pimiä R, Nohynek L, Meier C. Antimicrobial properties of phenolic compounds from berries. J Appl Microbiol 2001;90(4):494507.

3. Melini F, Melini V, Luziatelli F, Ficca AG, Ruzzi M. Health-Promoting Components in Fermented Foods: An Up-to-Date Systematic Review. Nutrients. 2019;11(5)

4. Szutowska J, Rybicka I, Pawlak-Lemańska K, Gwiazdowska D Spontaneously fermented curly kale juice: Microbiological quality, nutritional composition, antioxidant, and antimicrobial properties. J Food Sci 2020.

5. Organización Mundial de la salud. No Title. Resistencia a los antibioticos. 2020.

6. Calvo J, Martínez-Martínez L. Mecanismos de acción de los antimicrobianos. Enferm Infecc Microbiol Clin. 2009 Jan 1;27(1):4452.

7. Rajapaksha P, Elbourne A, Gangadoo S, Brown R, Cozzolino D, Chapman J. A review of methods for the detection of pathogenic microorganisms. Vol. 144, Analyst. Royal Society of Chemistry 2019: 396-411.

8. M. Farthing, M. Salam. Diarrea aguda en adultos y niños: una perspectiva mundial 2012.

9. Hill C, Guarner F, Reid G, Gibson GR, Merenstein DJ, Pot B, et al. The International Scientific Association for Probiotics and Prebiotics consensus statement on the scope and appropriate use of the term probiotic. Nat Rev Gastroenterol Hepatol 2014; 11:506-14.

10. Douillard FP, de Vos WM. Functional genomics of lactic acid bacteria: From food to health. Microb Cell Fact 2014 ;13(1):1-21.

11. Rivera JA, Rosas-Peralta OM-HM, Popkin CAA-SBM, Willett WC. Consumo de bebidas para una vida saludable: recomendaciones para la población mexicana 2008.

12. Dávila de Campagnaro E. Bebidas vegetales y leches de otros mamíferos. Arch Venez Pueric Pediatr. 2017;80(3):96-101.

13. Scholz-Ahrens KE, Ahrens F, Barth CA. Nutritional and health attributes of milk and milk imitations. Vol. 59, European Journal of Nutrition. Springer; 2020: 19-34.

14. Goldsmith PD. Economics of Soybean Production, Marketing, and Utilization. In: Soybeans: Chemistry, Production, Processing, and Utilization. Elsevier Inc.; 2008. p. 117-50.

15. Popkin BM, Armstrong LE, Bray GM, Caballero B, Frei B, Willett WC. A new proposed guidance system for beverage consumption in the United States. Vol. 83, American Journal of Clinical Nutrition. American Society for Nutrition; 2006: 529-42.

16. Blandino A, Al-Aseeri ME, Pandiella SS, Cantero D, Webb C. Cerealbased fermented foods and beverages. Vol. 36, Food Research International. Elsevier Ltd; 2003: 527-43.

17. Panghal A, Janghu S, Virkar K, Gat Y, Kumar V, Chhikara N. Potential non-dairy probiotic products - A healthy approach. Vol. 21, Food Bioscience. Elsevier Ltd; 2018: 80-9.

18. Mäkinen OE, Wanhalinna V, Zannini E, Arendt EK. Foods for Special Dietary Needs: Non-dairy Plant-based Milk Substitutes and Fermented Dairy-type Products. Crit Rev Food Sci Nutr 2016;56(3):339-49.

19. Singh D, Sharma RR. Postharvest Diseases of Fruits and Vegetables and Their Management. In: Postharvest Disinfection of Fruits and Vegetables. Elsevier; 2018: 1-52.

20. Pérdidas y desperdicios de alimentos en América Latina y el Caribe | FAO 2020.

21. Moñino M, Baladia E, Palou A, Russolillo G, Marques I, Farran A, et al. Consumption of fruit juices in the framework of a healthy diet: Position Paper of the Scientific Committee " 5 a day." Act Diet. 2010, $1 ; 14(3): 138-43$

22. Federación DO de la. NORMA Oficial Mexicana NOM-173-SCFI2009 , Jugos de frutas preenvasados-Denominaciones, especificaciones fisicoquímicas, información comercial y métodos de prueba 2009.

23. Albrecht C, Matias Scavuzzo C. Manual de frutas y hortalizas. Propiedades fisicoquímicas y condiciones de manipulación y conservación 2020.

24. Sahreen S, Khan MR, Khan RA. Comprehensive assessment of phenolics and antiradical potential of Rumex hastatus D. Don. roots. BMC Complement Altern Med 2014;14(1):1-11.

25. Rabie MA, Soliman AZ, Diaconeasa ZS, Constantin B. Effect of Pasteurization and Shelf Life on the Physicochemical Properties of Physalis ( $P$ hysalis peruviana L.) Juice. J Food Process Preserv 2015;39(6):1051-60. 
26. Vitale AA, Bernatene EA, Pomilio AB. Bioquímica Clínica Actualización Carotenoides en quimioprevención: Licopeno .Vol. 44, Acta Bioquím Clín Latinoam. 2010.

27. Mozos I, Stoian D, Caraba A, Malainer C, Horbanczuk JO, Atanasov AG. Lycopene and vascular health. Vol. 9, Frontiers in Pharmacology. Frontiers Media S.A. 2018: 521.

28. Meléndez-Martínez AJ, Mapelli-Brahm P, Stinco CM. The colourless carotenoids phytoene and phytofluene: From dietary sources to their usefulness for the functional foods and nutricosmetics industries. J Food Compos Anal. 2018, 1;67:91-103.

29. Al-Niaimi F, Zhen Chiang NY. Topical Vitamin C and the skin: Mechanisms of action and Clinical applications. Vol. 10, Journal of Clinical and Aesthetic Dermatology. Matrix Medical Communications 2017: 14-7.

30. Du J, Cullen JJ, Buettner GR. Ascorbic acid: Chemistry, biology and the treatment of cancer. Vol. 1826, Biochimica et Biophysica Acta Reviews on Cancer. Elsevier; 2012: 443-57.

31. Tönnies E, Trushina E. Oxidative Stress, Synaptic Dysfunction, and Alzheimer's Disease. Vol. 57, Journal of Alzheimer's Disease. IOS Press; 2017. 1105-21.

32. Moretti M, Fraga DB, Rodrigues ALS. Preventive and therapeutic potential of ascorbic acid in neurodegenerative diseases. CNS Neurosci Ther 2017: 23(12):921-9.

33. Scalbert A, Manach C, Morand C, Rémésy C, Jiménez L. Dietary polyphenols and the prevention of diseases. Vol. 45, Critical Reviews in Food Science and Nutrition. Taylor \& Francis Group 2005: 287 306.

34. Wu Y, Liu W, Li Q, Li Y, Yan Y, Huang F, et al. Dietary chlorogenic acid regulates gut microbiota, serum-free amino acids and colonic serotonin levels in growing pigs. Int J Food Sci Nutr 2018 ;69(5):56673.

35. Mena P, Bresciani L. Dietary fibre modifies gut microbiota: what's the role of (poly)phenols?. Vol. 71, International Journal of Food Sciences and Nutrition. Taylor and Francis Ltd.; 2020: 783-4.

36. Starek A, Pawłat J, Chudzik B, Kwiatkowski M, Terebun P, Sagan A, et al. Evaluation of selected microbial and physicochemical parameter of fresh tomato juice after cold atmospheric pressure plasma treatment during refrigerated storage. Sci Rep 2019; 9(1):1-11.

37. Jayathunge KGLR, Stratakos AC, Cregenzán-Albertia O, Grant IR Lyng J, Koidis A. Enhancing the lycopene in vitro bioaccessibility of tomato juice synergistically applying thermal and non-thermal processing technologies. Food Chem. 2017; 221:698-705.

38. Jafari SM, Jabari SS, Dehnad D, Shahidi SA. Effects of thermal processing by nanofluids on vitamin $\mathrm{C}$, total phenolics and total soluble solids of tomato juice. J Food Sci Technol 2017 ;54(3):679-86.

39. Samreen, Ch V.V. Satyanarayana LE, Beera V, Rao VS Physicochemical Characteristics of Pomegranate and Pineapple Juice. Indian J Ecol. 2020;(43):60-3.

40. Lagnika C, Adjovi YCS, Lagnika L, Gogohounga FO, Do-Sacramento O, Koulony RK, et al. Effect of Combining Ultrasound and Mild Hea Treatment on Physicochemical, Nutritional Quality and Microbiological Properties of Pineapple Juice. Food Nutr Sci 2017, 8(02):227-41.
41. Owolade SO, Akinrinola AO, Popoola FO, Aderibigbe, O. R., Ademoyegun, O. T. Olabode IA. Study on physico-chemical properties, antioxidant activity and shelf stability of carrot (Daucus carota) and pineapple (Ananas comosus) juice blend. Int Food Res J. 2017;24(2)

42. Adubofuor J, Amoah I, Ayivi R Dela. Effects of Blanching on Physicochemical Properties of Chantenay Carrots Juice and Assessing the Qualities of Formulated Carrot-MD2 Pineapple Juice Blends . Am J Food Sci Technol. 2016; 4(3):81-8

43. Simran A, Saleem S, Rakesh G. Physicochemical and Bioactive Compounds in Carrot and Beetroot Juice-Indian Journals. Asian J Dairy Food Res 2019; 38.

44. Pokhrel PR, Bermúdez-Aguirre D, Martínez-Flores HE, Garnica-Romo MG, Sablani S, Tang J, et al. Combined Effect of Ultrasound and Mild Temperatures on the Inactivation of E. coli in Fresh Carrot Juice and Changes on its Physicochemical Characteristics. J Food Sci. 2017 ;82(10):2343-50.

45. Stinco CM, Szczepańska J, Marszałek K, Pinto CA, Inácio RS, Mapelli-Brahm P, et al. Effect of high-pressure processing on carotenoids profile, colour, microbial and enzymatic stability of cloudy carrot juice. Food Chem. 2019

46. Porto M, Okina V, Pimentel T, Prudencio S. Physicochemical Stability, Antioxidant Activity, and Acceptance of Beet and Orange Mixed Juice During Refrigerated Storage. Beverages. 2017; 3(4):36.

47. Saeeduddin M, Abid M, Jabbar S, Wu T, Yuan Q, Riaz A, et al. Nutritional, microbial and physicochemical changes in pear juice under ultrasound and commercial pasteurization during storage. J Food Process Preserv 2017; 41(6)

48. Saeeduddin M, Abid M, Jabbar S, Hu B, Hashim MM, Khan MA, et al. Physicochemical parameters, bioactive compounds and microbial quality of sonicated pear juice. Int $\mathrm{J}$ Food Sci Technol. 2016;51(7):1552-9.

49. Shourove JH, Zzaman W, Chowdhury RS, Hoque MM. Effect of Thermal Treatment on Physicochemical Stability and Antioxidant Properties of Locally Available Underutilized Star Fruit Juice. Asian Food Sci J. 2020; 14(3):41-53.

50. Jerry A-A, Bright Q. Effect of storage temperature on the physicochemical, nutritional and microbiological quality of pasteurised soursop (Annona muricata L.) Juice. African J Food Sci 2019;13(2):38-47.

51. Ruiz-De Anda D, Ventura-Lara MG, Rodríguez-Hernández G, Ozuna C. The impact of power ultrasound application on physicochemical, antioxidant, and microbiological properties of fresh orange and celery juice blend. J Food Meas Charact. 2019; 13(4):3140-8.

52. Włodarska K, Pawlak-Lemańska K, Górecki T, Sikorska E. Perception of Apple Juice: A Comparison of Physicochemical Measurements, Descriptive Analysis and Consumer Responses. J Food Qual. 2016; 39(4):351-61

53. Włodarska K, Pawlak-Lemańska K, Sikorska E. Prediction of key sensory attributes of apple juices by multivariate analysis of their physicochemical profiles. Br Food J. 2019; 121(10):2429-41.

54. Sroy S, Fundo JF, Miller FA, Brandão TRS, Silva CLM. Impact of ozone processing on microbiological, physicochemical, and bioactive characteristics of refrigerated stored Cantaloupe melon juice. J Food Process Preserv. 2019;43(12). 
55. Pagliarulo C, De Vito V, Picariello G, Colicchio R, Pastore G, Salvatore $\mathrm{P}$, et al. Inhibitory effect of pomegranate (Punica granatum L.) polyphenol extracts on the bacterial growth and survival of clinical isolates of pathogenic Staphylococcus aureus and Escherichia coli. Food Chem. 2016; 190:824-31.

56. Singh JP, Kaur A, Singh N, Nim L, Shevkani K, Kaur H, et al. In vitro antioxidant and antimicrobial properties of jambolan (Syzygium cumini) fruit polyphenols. LWT. 2016; 65:1025-30.

57. Burdulis D, Šarkinas A, Jasutiene I, Stackevičiene E, Nikolajevas L, Janulis V. Comparative study of anthocyanin composition, antimicrobial and antioxidant activity in bilberry (Vaccinium myrtillus L.) and blueberry (Vaccinium corymbosum L.) fruits. Acta Pol Pharm - Drug Res. 2009; 66(4):399-408.

58. Nereyda E, Sauceda R. Uso de agentes antimicrobianos naturales en la conservación de frutas y hortalizas. Vol. 7.

59. Lima VN, Oliveira-Tintino CDM, Santos ES, Morais LP, Tintino SR Freitas TS, et al. Antimicrobial and enhancement of the antibiotic activity by phenolic compounds: Gallic acid, caffeic acid and pyrogallol. Microb Pathog. 2016; 99:56-61.

60. Mason TL, Bruce P. W. Inactivation of red beet $\beta$-glucan synthase by native and oxidized phenolic compounds. Phytochemistry. 1987; 26(8):2197-202

61. Ulanowska K, Tkaczyk A, Konopa G, Wȩgrzyn G. Differential antibacterial activity of genistein arising from global inhibition of DNA, RNA and protein synthesis in some bacterial strains. Arch Microbiol. 2006; 184(5):271-8.

62. Eumkeb G, Chukrathok S. Synergistic activity and mechanism of action of ceftazidime and apigenin combination against ceftazidimeresistant Enterobacter cloacae. Phytomedicine. 2013;20(3-4):262-9.

63. Cushnie TPT, Lamb AJ. Detection of galangin-induced cytoplasmic membrane damage in Staphylococcus aureus by measuring potassium loss. J Ethnopharmacol. 2005; 101(1-3):243-8.

64. Cushnie TPT, Lamb AJ. Recent advances in understanding the antibacterial properties of flavonoids. Vol. 38, International Journal of Antimicrobial Agents. Elsevier B.V.; 2011: 99-107.

65. Simões CC, De Araújo DB, De Araújo RPC. Study, in vitro and ex vivo, of the action of different concentrations of propolis extracts against microorganisms present in human saliva. Brazilian J Pharmacogn. 2008; 18(1):84-9.

66. Oikeh EI, Omoregie ES, Oviasogie FE, Oriakhi K. Phytochemical, antimicrobial, and antioxidant activities of different citrus juice concentrates. Food Sci Nutr. 2016; 4(1):103-9.

67. Barberis A, Deiana M, Spissu Y, Azara E, Fadda A, Serra PA, et al. Antioxidant, Antimicrobial, and Other Biological Properties of Pompia Juice. Molecules. 2020; 25(14):3186.

68. Fessard A, Kapoor A, Patche J, Assemat S, Hoarau M, Bourdon E, et al. Lactic Fermentation as an Efficient Tool to Enhance the Antioxidan Activity of Tropical Fruit Juices and Teas. Microorganisms. 2017; $5(2): 23$

69. Puerta QGI. Fundamentos del proceso de fermentación en el beneficio del café. Fed Nac Cafe Colomb. 2010.

70. Frias J, Martinez-Villaluenga C, Peñas E. Fermented foods in health and disease prevention. Elsevier; 2017.
71. Vilela A. The Importance of Yeasts on Fermentation Quality and Human Health-Promoting Compounds. Fermentation. 2019; 5(2):46.

72. Mishra SS, Ray RC, Panda SK, Montet D. Technological Innovations in Processing of Fermented Foods. An Overview. In: Ray RC, Montet D, editors. In Fermented Foods, Part II: Technological Intervention. 1st ed. Taylors and Francis, CRC; 2017: 525.

73. Ray RC, Joshi V. Fermented Foods: Past, present, and future scenario. In: Ray RC, Montet D, editors. Microorganisms and Fermentation of Traditional Foods. CRC Press; 2014: 1-36.

74. Salminen S, Von Wright A. Lactic Acid Bacteria. New York: Dekker; 1993: 199-225.

75. Smit G, Smit BA, Engels WJM. Flavour formation by lactic acid bacteria and biochemical flavour profiling of cheese products. FEMS Microbiol Rev. 2005;29(3):591-610.

76. Kabak B, Dobson ADW. An introduction to the traditional fermented foods and beverages of Turkey. Crit Rev Food Sci Nutr. 2011;51(3):248-60.

77. Wu C, Huang J, Zhou R. Genomics of lactic acid bacteria: Current status and potential applications. Vol. 43, Critical Reviews in Microbiology. Taylor and Francis Ltd; 2017: 393-404.

78. Rattanachaikunsopon P, Phumkhachorn P. Lactic acid bacteria: their antimicrobial compounds and their uses in food production. Ann Biol Res. 2010; 1:218-28.

79. Bintsis T. Lactic acid bacteria as starter cultures: An update in their metabolism and genetics. AIMS Microbiol. 2018;4(4):665-84.

80. Vodnar DC, Paucean A, Dulf FV, Socaciu C. HPLC characterization of lactic acid formation and FTIR fingerprint of probiotic bacteria during fermentation processes. Not Bot Horti Agrobot Cluj-Napoca. 2010; 38(2):109-13

81. Lee KW, Shim JM, Park SK, Heo HJ, Kim HJ, Ham KS, et al. Isolation of lactic acid bacteria with probiotic potentials from kimchi, traditional Korean fermented vegetable. LWT - Food Sci Technol. 2016; 71:1307.

82. Abushelaibi A, Al-Mahadin S, El-Tarabily K, Shah NP, Ayyash M. Characterization of potential probiotic lactic acid bacteria isolated from camel milk. LWT - Food Sci Technol. 2017 ;79:316-25.

83. Taroub B, Salma L, Manel Z, Ouzari HI, Hamdi Z, Moktar H. Isolation of lactic acid bacteria from grape fruit: antifungal activities, probiotic properties, and in vitro detoxification of ochratoxin A. Ann Microbiol. 2019; 69(1):17-27.

84. Verón HE, Di Risio HD, Isla MI, Torres S. Isolation and selection of potential probiotic lactic acid bacteria from Opuntia ficus-indica fruits that grow in Northwest Argentina. LWT - Food Sci Technol. 2017; $84: 231-40$.

85. Fessard A, Remize F. Genetic and technological characterization of lactic acid bacteria isolated from tropically grown fruits and vegetables. Int J Food Microbiol. 2019;301:61-72.

86. Ricci A, Cirlini M, Calani L, Bernini V, Neviani E, Del Rio D, et al. In vitro metabolism of elderberry juice polyphenols by lactic acid bacteria. Food Chem. 2019; 276:692-9.

87. Oh NS, Joung JY, Lee JY, Kim Y. Probiotic and anti-inflammatory potential of Lactobacillus rhamnosus 4B15 and Lactobacillus gasseri 
4M13 isolated from infant feces. Forestier C, editor. PLoS One. 2018; 13(2).

88. Hayek SA, Ibrahim SA. Current Limitations and Challenges with Lactic Acid Baceria: A Review. Food Nutrtion Sci. 2013;4:73-87.

89. Valdovinos MA, Montijo E, Abreu AT, Heller S, González-Garay A, Bacarreza D, et al. Consenso mexicano sobre probióticos en gastroenterología. Vol. 82, Revista de Gastroenterologia de Mexico. Asociacion Mexicana de Gastroenterologia; 2017. p. 156-78.

90. König H, Fröhlich J. Lactic acid bacteria. In: Biology of Microorganisms on Grapes, in Must and in Wine. Springer International Publishing; 2017: 3-41.

91. Özcelik S, Kuley E, Özogul F. Formation of lactic, acetic, succinic, propionic, formic and butyric acid by lactic acid bacteria. LWT - Food Sci Technol. 2016; 73:536-42.

92. Tarrah A, De Castilhos J, Rossi RC, Da Silva Duarte V, Ziegler DR, Corich V, et al. In vitro probiotic potential and anti-cancer activity of newly isolated folate-producing streptococcus thermophilus strains. Front Microbiol. 2018;9.

93. Bautista-Gallego J, Ferrocino I, Botta C, Ercolini D, Cocolin L, Rantsiou K. Probiotic potential of a Lactobacillus rhamnosus cheese isolate and its effect on the fecal microbiota of healthy volunteers. Food Res Int. 2019;119:305-14.

94. Pessione E, Cirrincione S. Bioactive molecules released in food by lactic acid bacteria: Encrypted peptides and biogenic amines. Front Microbiol. 2016;7.

95. Bujna E, Farkas NA, Tran AM, Dam MS, Nguyen QD. Lactic acid fermentation of apricot juice by mono- and mixed cultures of probiotic Lactobacillus and Bifidobacterium strains. Food Sci Biotechnol. 2018;27(2):547-54.

96. Ray RC. Microbial Biotechnology in Horticulture. CRC Press; 2006.

97. Chwastek A, Klewicka E, Klewicki R, Sójka M. Lactic Acid Fermentation of Red Beet Juice Supplemented with Waste Highbush Blueberry-Sucrose Osmotic Syrup as a Method of Probiotic Beverage Production. J Food Process Preserv. 2016 ;40(4):780-9.

98. Costa MGM, Fonteles TV, De Jesus ALT, Rodrigues S. Sonicated pineapple juice as substrate for $\mathrm{L}$. casei cultivation for probiotic beverage development: Process optimisation and product stability. Food Chem. 2013;139(1-4):261-6.

99. Chen H, Yu W, Chen G, Meng S, Xiang Z, He N. Antinociceptive and antibacterial properties of anthocyanins and flavonols from fruits of black and non-black mulberries. Molecules. 2018;23(1).

100. Cai W, Tang F, Zhao X, Guo Z, Zhang Z, Dong Y, et al. Differen lactic acid bacteria strains affecting the flavor profile of fermented jujube juice. J Food Process Preserv. 2019 ;43(9).

101. Liao XY, Guo LQ, Ye ZW, Qiu LY, Gu FW, Lin JF. Use of autochthonous lactic acid bacteria starters to ferment mango juice for promoting its probiotic roles. Prep Biochem Biotechnol. 2016; 46(4):399-405.

102. Ricci A, Cirlini M, Levante A, Dall'Asta C, Galaverna G, Lazzi C. Volatile profile of elderberry juice: Effect of lactic acid fermentation using L. plantarum, L. rhamnosus and L. casei strains. Food Res Int. 2018; 105:412-22.
103. Kaprasob R, Kerdchoechuen O, Laohakunjit N, Sarkar D, Shetty K. Fermentation-based biotransformation of bioactive phenolics and volatile compounds from cashew apple juice by select lactic acid bacteria. Process Biochem. 2017; 59:141-9.

104. Verón HE, Gauffin Cano P, Fabersani E, Sanz Y, Isla MI, Fernández Espinar MT, et al. Cactus pear (Opuntia ficus-indica) juice fermented with autochthonous Lactobacillus plantarum S-811. Food Funct. 2019;10(2):1085-97.

105. Ricci A, Cirlini M, Maoloni A, Del Rio D, Calani L, Bernini V, et al. Use of dairy and plant-derived lactobacilli as starters for cherry juice fermentation. Nutrients. 2019;11(2).

106. Kwaw E, Ma Y, Tchabo W, Apaliya MT, Wu M, Sackey AS, et al. Effect of lactobacillus strains on phenolic profile, color attributes and antioxidant activities of lactic-acid-fermented mulberry juice. Food Chem. 2018;250:148-54.

107. Neffe-Skocińska K, Rzepkowska A, Szydłowska A, KołozynKrajewska D. Trends and Possibilities of the Use of Probiotics in Food Production. Altern Replace Foods. 2018;17:65-94.

108. Vaithilingam M, Chandrasekaran S, Mehra A, Prakash S, Agarwal A, Ethiraj S, et al. Fermentation of Beet Juice Using Lactic Acid Bacteria and its Cytotoxic Activity Against Human Liver Cancer Cell Lines HepG2. 2016

109. Reid G, Jass J, Sebulsky MT, McCormick JK. Potential Uses of Probiotics in Clinical Practice. Clin Microbiol Rev. 2003 ;16(4):65872.

110. Wuyts S, Van Beeck W, Oerlemans EFM, Wittouck S, Claes IJJ, De Boeck I, et al. Carrot juice fermentations as man-made microbial ecosystems dominated by lactic acid bacteria. Appl Environ Microbiol. 2018; 84(12):134-52.

111. Ayed L, Ben Abid S, Hamdi M. Development of a beverage from red grape juice fermented with the Kombucha consortium. Ann Microbiol. 2017; 67(1):111-21

112. Michalak M, Kubik-Komar A, Waśko A, Polak-Berecka M. Starter culture for curly kale juice fermentation selected using principal component analysis. Food Biosci. 2020, 35.

113. Thakur A, Joshi VK. Preparation of Probiotic Apple Juice by Lactic Acid Fermentation. Int J Food Ferment Technol. 2017;7(1):67.

114. Rolfe RD. The Role of Probiotic Cultures in the Control of Gastrointestinal Health. J Nutr. 2000;130(2):396S-402S.

115. Djadouni F. Scientia Agriculturae Characterization Of Bacteriocin Produced By Lactobacillus Brevis Isilated From Traditional Fermented Tomatoes Juice Use of biocteriocins in biotherapy View project Food biopreservation by use bacteriocins View project. 2016

116. Guinane CM, Piper C, Draper LA, O’Connor PM, Hill C, Paul Ross $\mathrm{R}$, et al. Impact of environmental factors on bacteriocin promoter activity in gut-derived Lactobacillus salivarius. Appl Environ Microbiol. 2015; 81(22):7851-9.

117. Kommineni S, Bretl DJ, Lam V, Chakraborty R, Hayward M, Simpson $\mathrm{P}$, et al. Bacteriocin production augments niche competition by enterococci in the mammalian gastrointestinal tract. Nature. 2015; 526(7575):719-22.

118. Sonbol FI, Abdel Aziz AA, El-Banna TE, Al-Fakhrany OM. Antimicrobial activity of bacteriocins produced by Enterococcus 
isolates recovered from Egyptian homemade dairy products against some foodborne pathogens. Int Microbiol. 2020;23(4):533-47.

119. Devi SM, Halami PM. Detection and characterization of pediocin PA1/AcH like bacteriocin producing lactic acid bacteria. Curr Microbiol. 2011;63(2):181-5.

120. Martinez RCR, De Martinis ECP. Evaluation of bacteriocin-producing Lactobacillus sakei 1 against Listeria monocytogenes $1 / 2$ a growth and haemolytic activity. Brazilian J Microbiol. 2005; 36(1):83-7.

121. Gupta S, Pandey S. Isolation and Characterization of Bacteriocin Producing Bacteria from Sweet Lime Juice. J PurE Appl Microbiol. 2018; 12(2):953-60.

122. Tenea *, Barrigas GN. The efficacy of bacteriocin-containing cell-free supernatant from Lactobacillus plantarum Cys5-4 to control pathogenic bacteria growth in artisanal beverages. Vol. 25, International Food Research Journal. 2018.

123. Pisano MB, Fadda ME, Melis R, Ciusa ML, Viale S, Deplano M, et al Molecular identification of bacteriocins produced by Lactococcus lactis dairy strains and their technological and genotypic characterization. Food Control. 2015; 51:1-8.

124. García-Cayuela T, Requena T, Martínez-Cuesta MC, Peláez C. Rapid detection of Lactococcus lactis isolates producing the lantibiotics nisin, lacticin 481 and lacticin 3147 using MALDI-TOF MS. J Microbiol Methods. 2017; 139:138-42.

125. Prosekov A, Babich O, Ivanova S, Pavsky VA. Article in Progress in Nutrition. 2017.

126. Heredia-Castro P, Hérnández-Mendoza A, González-Córdova A Vallejo-Cordoba B. Bacteriocinas de bacterias ácido lácticas: mecanismos de acción y actividad antimicrobiana contra patógenos en queso. Asoc Interciencia. 2017; 42(6):340-6

127. Yusuf M, Haziyamin T, Hamid TA. Lactic Acid Bacteria:Bacteriocin Producer: A Mini Review . Artic IOSR J Pharm. 2013.

128. Flemer B, Warren RD, Barrett MP, Cisek K, Das A, Jeffery IB, et al. The oral microbiota in colorectal cancer is distinctive and predictive. Gut. 2018; 67(8):1454-63.

129. Arqués JL, Rodríguez E, Langa S, Landete JM, Medina M Antimicrobial activity of lactic acid bacteria in dairy products and gut: Effect on pathogens. Biomed Res Int. 2015.

130. Thaiss CA, Zmora N, Levy M, Elinav E. The microbiome and innate immunity. Vol. 535, Nature. Nature Publishing Group; 2016: 65-74.

131. Sekirov I, Russell SL, Caetano M Antunes L, Finlay BB. Gu microbiota in health and disease [Internet]. Vol. 90, Physiological Reviews. American Physiological Society Bethesda, MD; 2010: 859904.

132. Ramakrishna BS. The Normal Bacterial Flora of the Human Intestine and Its Regulation. J Clin Gastroenterol. 2007; 41.

133. Kamada T, Haruma K, Ito M, Inoue K, Manabe N, Matsumoto H, et al. Time Trends in Helicobacter pylori Infection and Atrophic Gastritis Over 40 Years in Japan. Helicobacter. 2015; 20(3):192-8.

134. Stecher B, Hardt WD. The role of microbiota in infectious disease Trends Microbiol. 2008; 16(3):107-14.
135. Kafshdooz T, Akbarzadeh A, Majdi Seghinsara A, Pourhassan M, Nasrabadi HT, Milani M. Role of Probiotics in Managing of Helicobacter Pylori Infection: A Review. Drug Res (Stuttg). 2017;67(2):88-93.

136. Espinoza DB, Guerrero CJ, Mayer PM. Nutrición Hospitalaria Hipótesis alternativas sobre los beneficios de los fermentados sobre la microbiota intestinal. 2018

137. Campana R, Van Hemert S, Baffone W. Strain-specific probiotic properties of lactic acid bacteria and their interference with human intestinal pathogens invasion. Gut Pathog. 2017; 9(1):12.

138. Hertzberger R, Arents J, Dekker HL, Pridmore RD, Gysler C, Kleerebezem M, et al. $\mathrm{H} 2 \mathrm{O} 2$ production in species of the Lactobacillus acidophilus group: A central role for a novel NADH-dependent flavin reductase. Appl Environ Microbiol. 2014; 80(7):2229-39.

139. Klewicka E, Zduńczyk Z, Juśkiewicz J. Effect of lactobacillus fermented beetroot juice on composition and activity of cecal microflora of rats. Eur Food Res Technol. 2009; 229(1):153-7.

140. Klewicka E, Zduńczyk Z, Juśkiewicz J, Klewicki R. Effects of Lactofermented Beetroot Juice Alone or with N-nitroso-N-methylurea on Selected Metabolic Parameters, Composition of the Microbiota Adhering to the Gut Epithelium and Antioxidant Status of Rats. Nutrients. 2015; 7(7):5905-15.

141. Cheng Y, Wu T, Chu X, Tang S, Cao W, Liang F, et al. Fermented blueberry pomace with antioxidant properties improves fecal microbiota community structure and short chain fatty acids production in an in vitro mode. LWT. 2020 